\title{
24-Hour Recording of Parkinsonian Gait Using a Portable Gait Rhythmogram
}

\author{
Hiroshi Mitoma $^{1}$, Mitsuru Yoneyama ${ }^{2}$ and Satoshi Orimo ${ }^{3}$
}

\begin{abstract}
Objective In the advanced stage of Parkinson's disease (PD), motor fluctuation is a frequent and a disabling problem. Despite its importance, motor fluctuation has received little scientific analysis probably due to limitation in objective assessment. Here, we focused on gait disorders to estimate motor fluctuation in daily activities.

Patients and Methods Using a new device, the portable gait rhythmogram, we recorded gait rhythm continuously over 24 hours in 22 patients with PD and in 11 normal controls, for quantitative evaluation of motor fluctuation. The duration of one gait cycle was measured.

Results Continuous 24-hour recording identified changes in gait rhythm, which correlated with fluctuation of PD symptoms. Different motor fluctuations were observed; a shift to a faster gait cycle was noted in patients with short-step walking, festination or freezing of gait, whereas a shift to a slower gait cycle was observed in patients with bradykinesia or instability.

Conclusion Characterization of motor fluctuation using this device could help in the selection of appropriate anti-PD medications.
\end{abstract}

Key words: Parkinson's disease, gait disorder, motor fluctuation

(Intern Med 49: 2401-2408, 2010)

(DOI: 10.2169/internalmedicine.49.3511)

\section{Introduction}

Dopamine-replacement therapy at the early stage of Parkinson's disease (PD) can improve motor and non-motor complications. In the advanced stage of the disease, however, motor fluctuation is a frequent and disabling problem. Up to $50 \%$ of patients exhibit motor fluctuation and unresponsiveness to medication after the first 5 years of treatment $(1,2)$. Despite its importance, motor fluctuation has received little scientific analysis probably due to the limitation in objective assessment since most evaluations depend on the patient's response to subjective historical questionnaires $(3,4)$.

Devices suitable for long-term monitoring of PD symptoms have been developed recently to detect on (i.e., effectively medicated)-off changes in the parkinsonian state. Wrist- or belt-mounted accelerometers (5-8) or six tri-axial accelerometers (9) have been used to monitor the degree of akinesia. Although the measurements of acceleration can provide a rough estimate of the quantity of movements, the nature of the executed movements remains unclear.

We focused on gait disorders in PD since analysis of gait disorders has the following two advantages. First, gait disorder is one of the cardinal symptoms of PD; disability of locomotion reduces the quality of daily life. Second, parkinsonian gait disorder is composed of composite elements, including freezing of gait, gait bradykinesia and postural instability (10-15). Due to such complexity, gait disorder reflects important pathomechanisms underlying PD and is therefore a good model for quantitative estimation. Recently, Moore et al (16) developed a system for the long-term monitoring of gait in PD, in which stride is calculated from the vertical linear acceleration. On the other hand, we analyzed gait rhythm in daily living activities. For example, inability to generate appropriate stride results in a compensatory in-

${ }^{1}$ Department of Medical Education, Tokyo Medical University, Tokyo, ${ }^{2}$ Mitsubishi Chemical Group Science and Technology Research Center, Inc., Tokyo and ${ }^{3}$ Department of Neurology, Kanto Chuo Hospital, Tokyo

Received for publication February 9, 2010; Accepted for publication August 9, 2010

Correspondence to Hiroshi Mitoma, mitoma@tokyo-med.ac.jp 

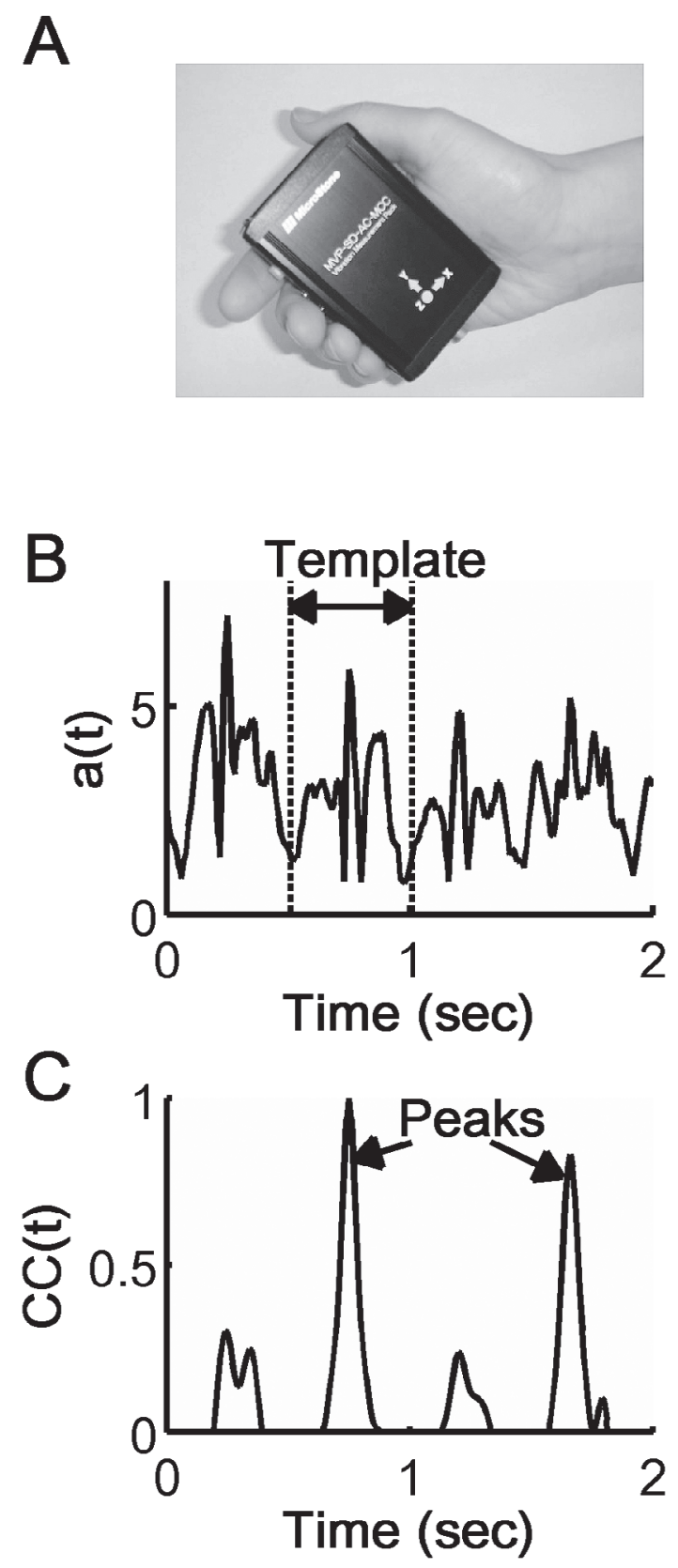

Figure 1. (A) The portable gait rhythmogram device. (B) Schematic representation of acceleration time series $(a(t))$, and a template for cross-correlation calculation. (C) Schematic representation of cross-correlation time series $(C C(t))$.

crease in cadence $(12,13)$, whereas bradykinesia forces the patient to walk slowly. Thus, continuous monitoring of gait rhythm could be utilized for screening PD status-related motor fluctuation. For this reason, we developed a portable device to continuously monitor gait rhythm and the duration of one gait cycle over a period of more than 24 hours, thus allowing quantitative estimation of motor fluctuation in daily activities.

\section{Patients and Methods}

\section{Measurements}

The portable gait rhythmogram (PGR) is a small device (size, $8 \times 6 \times 2 \mathrm{~cm}$, weight, $80 \mathrm{~g}$, Fig. 1A) that threedimensionally measures $\left(a_{\mathrm{x}}, a_{\mathrm{y}}, a_{\mathrm{z}}\right)$ the acceleration in trunk movements caused by step-in and kick-off, using accelerometers. The PGR is attached to the waist of the patient, and records the above signals at a sampling rate of $10 \mathrm{msec}$. The data are automatically stored in a micro SD card. When recording is completed, the absolute value of the acceleration vectors $\left(a ; a^{2}=a_{\mathrm{x}}{ }^{2}+a_{\mathrm{y}}{ }^{2}+a_{\mathrm{z}}{ }^{2}\right)$ is calculated off-line and graphically displayed on the PC (Fig. 2A, 3A). Furthermore, the peak-to-peak interval is automatically detected to calculate the duration of one gait cycle. Thus, this recording system measures both the amplitude of the gait acceleration and the gait cycle but not the stride length.

The acceleration vectors related to stepping can be distinguished from those related to other movements, since the former has steep curves and appears rhythmically. Furthermore, based on the mathematical method of 'pattern matching', we distinguished the acceleration vectors caused by stepping from those by other trunk and limb movements. First, attention was focused on relatively strong signal region (e.g., $a>1 \mathrm{~m} / \mathrm{s}^{2}$ ) in the acceleration time series, and a three-dimensional template wave $\left(a_{\mathrm{x}}, a_{\mathrm{y}}, a_{\mathrm{z}}\right)$ with a duration of about $0.5 \mathrm{sec}$ was arbitrarily chosen around a local maximum point from that region (Fig. 1B). Then, the crosscorrelation $C C(t)$ was calculated between this wave and the whole time series at each time $t$ using the following formula:

$$
C C(t)=\frac{\frac{1}{p} \sum_{i=1}^{p}\left[a_{x}(i) a_{x}(i+t)+a_{y}(i) a_{y}(i+t)+a_{z}(i) a_{z}(i+t)\right]}{\left\{\frac{1}{p} \sum_{i=1}^{p}\left[a_{x}(i)^{2}+a_{y}(i)^{2}+a_{z}(i)^{2}\right]\right\}^{\frac{1}{2}}\left\{\frac{1}{p} \sum_{i=1}^{p}\left[a_{x}(i+t)^{2}+a_{y}(i+t)^{2}+a_{z}(i+t)^{2}\right]\right\}^{\frac{1}{2}}}
$$

where $p$ is the length of the template wave. The obtained cross-correlation is a scalar time series, showing pronounced rhythmic peaks even when the initial signal is too noisy to visualize periodicity with the naked eye. If the acceleration change is caused by gait motion, the $C C(t)$ peaks exhibit alternate changes in magnitude with time due to left/right body sway during walking. Thus, we can pick up correct peaks corresponding to one gait cycle (Fig. 1C). This "pattern matching method" also enabled us to distinguish the acceleration vectors caused by stepping from unexpected and large artifacts. Moreover, even though the PGR moved from the original position during the recording and the form of the acceleration vectors changed, we were able to identify the acceleration vectors continuously.

A fully-charged PGR can achieve 40 consecutive hours of recording. In quantitative analysis, the duration of the gait cycle represents the mean value of the cycles recorded every 

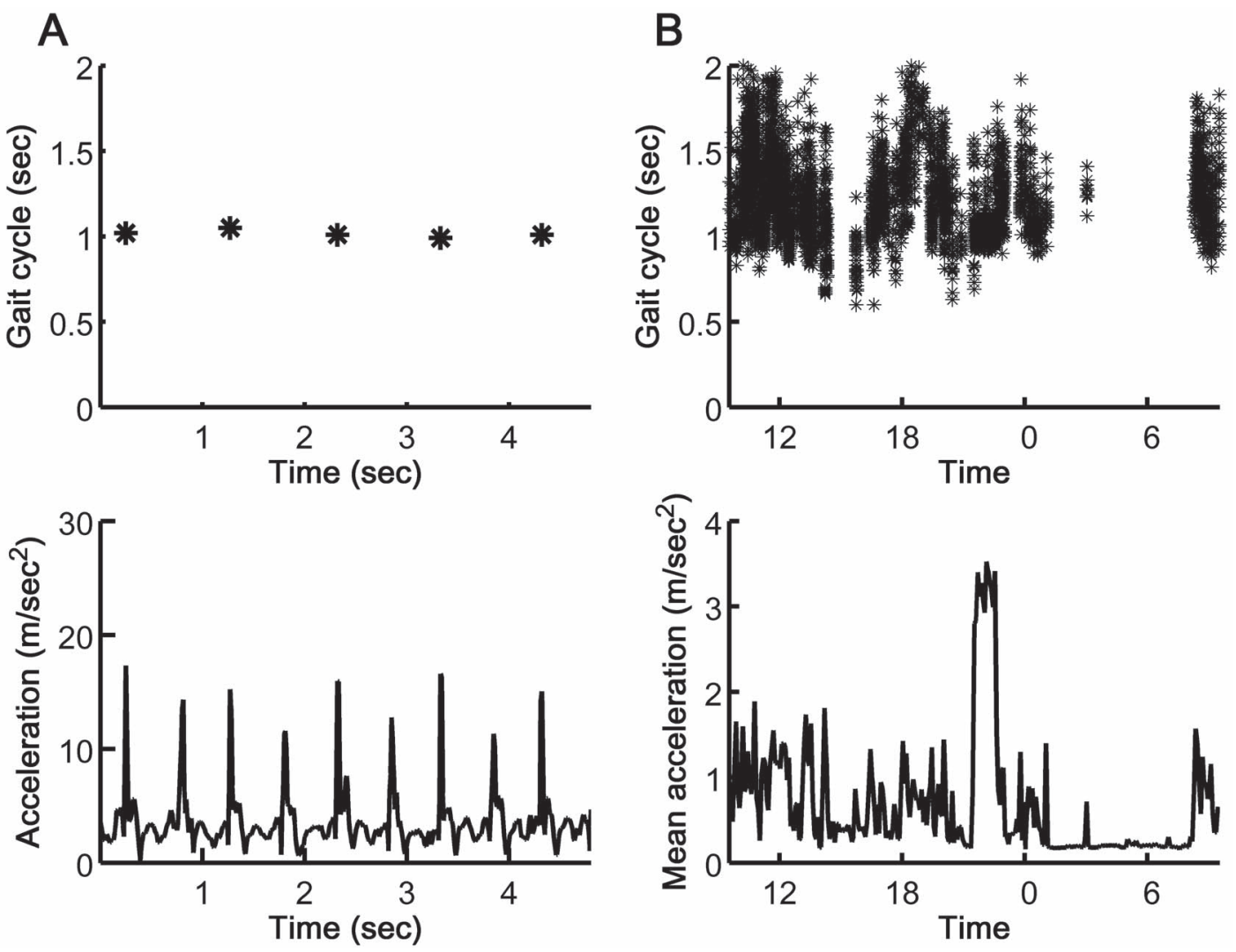

Figure 2. An example of continuous recording in a normal subject. (A) Reconstructed absolute values of acceleration vectors associated with gait (bottom) and plots of gait cycle duration calculated from their peak values (top). (B) The gait cycle duration of all steps during $24 \mathrm{hr}$ (top) and the absolute values of accelerations induced by all movements, including gait and limb/trunk movements, during $24 \mathrm{hr}$ (bottom).

hour. Data are excluded if the number of steps is less than 20 per hour. Values are presented as mean \pm SD.

\section{Subjects}

Using the PGR, we recorded continuous daily profiles of 22 PD patients (27 recordings) with a mean age of $68.2 \pm$ 11.3 years ( \pm SD, 9 men and 13 women), and 11 age- and height-matched normal controls (age, 68.3 \pm 16.1 years, 5 men and 6 women). Only PD patients who could walk unaided were studied; they included 1 patient with Hoehn and Yahr stage I, 8 with stage II, 8 with stage III, and 5 with stage IV. Motor fluctuation was observed in 10 patients. The clinical status was examined using the Unified Parkinson's Disease Rating Scale (UPDRS) motor score 'on' state.

Informed consent was obtained from all subjects. All procedures were conducted in accordance with the guidelines of the Ethics Committee of our institution.

\section{Results}

\section{4-hour recording}

Figure 2 shows an example of 24-hour recording in a control subject (a 68-year-old woman). The recording shows daily activities of a normal subject throughout the 24-hour (bottom graph, Fig. 2B), and the gait cycle duration of all steps during daily activities (top graph, Fig. 2B). The activities in the bottom graph, together with the patient's diary of daily activity, provide an index of the daily profile. Housekeeping activity was conducted throughout the morning and afternoon, represented by the high activity on the record. Especially, she fast-walked between 2200 and 2300. Furthermore, she slept from 0100 to 0800 , during which the activity was extremely low. At 0300 , however, she went to the lavatory, as evident from the activity on the record. The high daily activity means that the patient walked actively within the house throughout the day. Between 1400 and 1500, she did not walk but sat to rest in the living room. Even during such rest period, low activities induced by limb and trunk movements were recorded, which was different from the sleep-related recording. Although the duration of one gait cycle varied within a range of 0.8-1.8 seconds depending on daily activity, the mean value computed for every hour was almost constant throughout the day. The mean duration of one gait cycle computed from data of 11 control subjects was $1.10 \pm 0.075$ seconds.

Figure 3 is an example of 24-hour recording in a PD patient (Patient 6, a 63-year-old man, UPDRS motor score 'on' state 36 ). The daily activities were poor compared with the normal controls (Fig. 3B, bottom). The time for activi- 

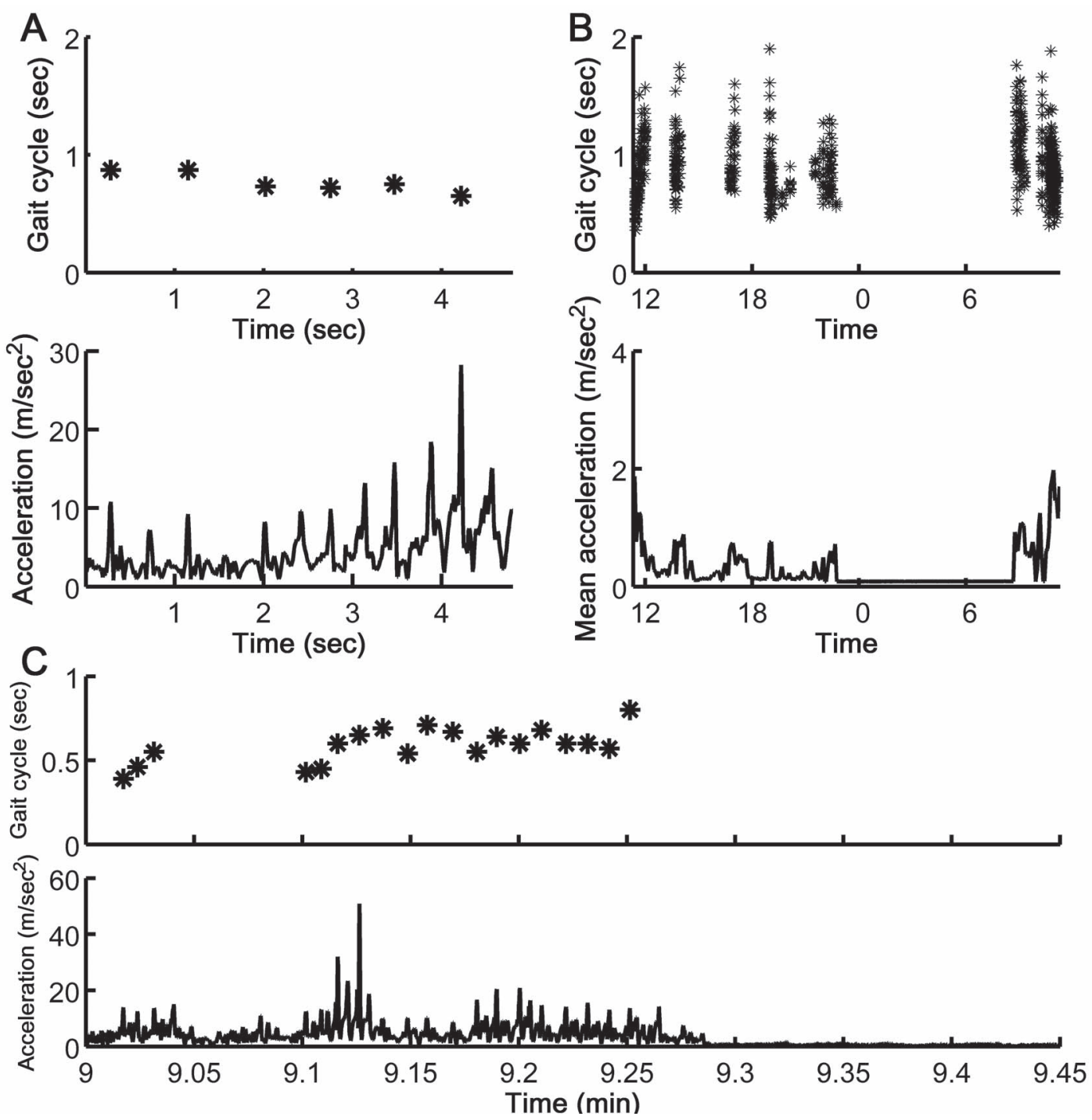

Figure 3. An Example of continuous recordings in a representative PD patient with UPDRS 'motor' 36. (A) Reconstructed absolute values of acceleration vectors associated with gait (bottom) and plots of gait cycle duration calculated from their peak values (top). (B) Gait cycle duration of all steps during $24 \mathrm{hr}$ (top) and the absolute values of accelerations induced by all movements, including gait and limb/trunk movements, during $24 \mathrm{hr}$ (bottom). (C) A recording of freezing of gait. Reconstructed absolute values in acceleration vectors associated with gait (bottom) and plots of gait cycle duration calculated from their peak values (top). The abscissa represents time from the beginning of the recording in this patient $(\mathrm{min})$.

ties decreased markedly and the amplitude of acceleration, induced by all movements, was low. Furthermore, zero activity, representing complete lack of activity, was observed frequently. The recording is suggestive of akinesia in this patient, i.e., poverty of movement. Correspondingly, the time spent walking was markedly less throughout the day compared with the control subjects (Fig. 3B, top).

A few minutes after wearing the PGR in the hospital, the patient accidentally exhibited festination and freezing of gait (FOG). Compared with the PGR recording of the normal control (Fig. 2A), the patient (Fig. 3A) exhibited festination, which is characterized by gradual shortening of the duration of one gait cycle, accompanied by increased acceleration. On the other hand, the time course of FOG is shown in
Fig. 3C. At the initiation of gait (9 minutes after the beginning of the recording), the patient showed an abrupt burst of shuffling trembling, which was recorded in a series of accelerations with high frequency (gait cycle duration of around 0.5 seconds). The trembling, that did not produce propelling forces, lasted about 15 seconds and ceased at the point of 9.3 minutes.

\section{Quantitative estimation of motor fluctuation}

Figure 4A shows changes in the duration of one gait cycle during walking in normal controls. During daily activi-

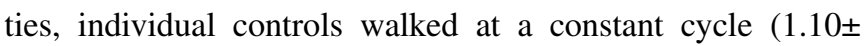
0.075 seconds). Thus, we characterized motor fluctuation subtypes based on the PGR-24-hour recording (Fig. 4B-D). 

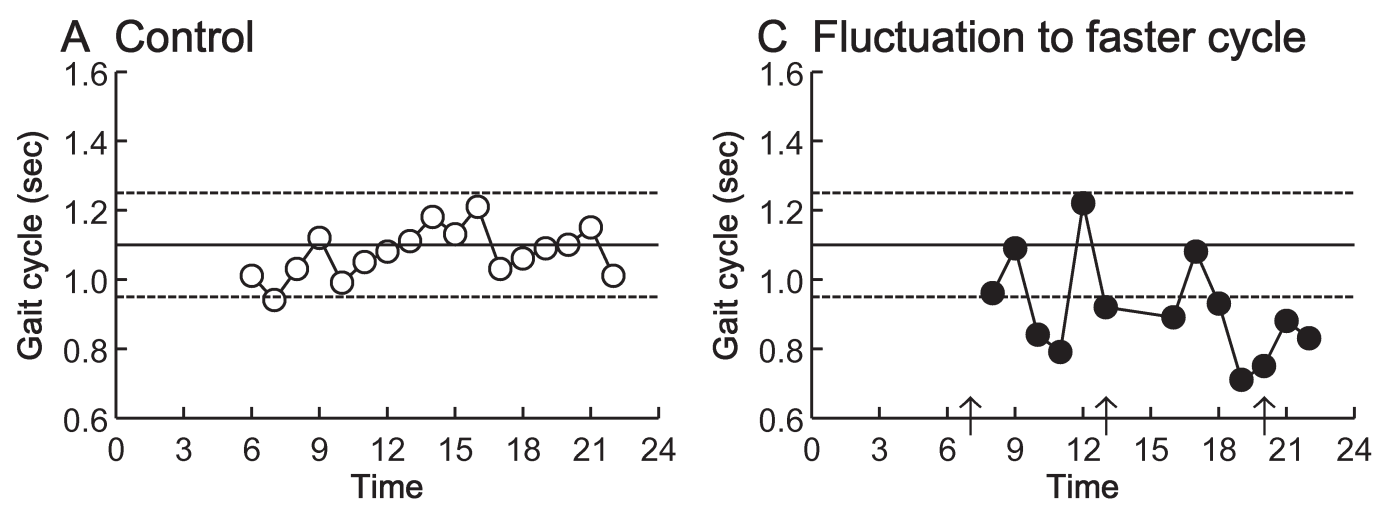

\section{B Well-controlled}

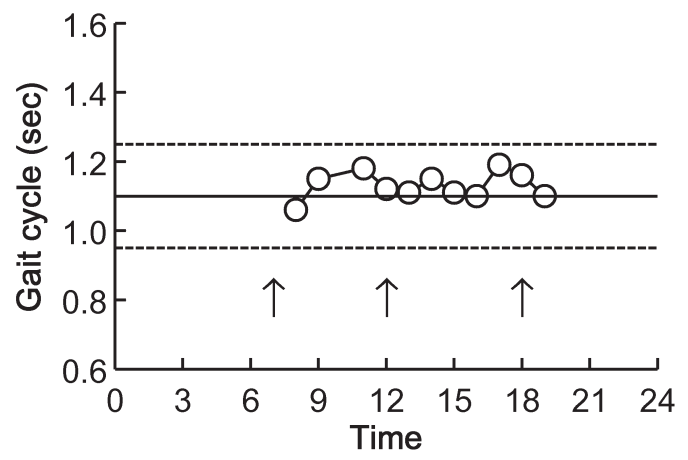

\section{Fluctuation to slower cycle}

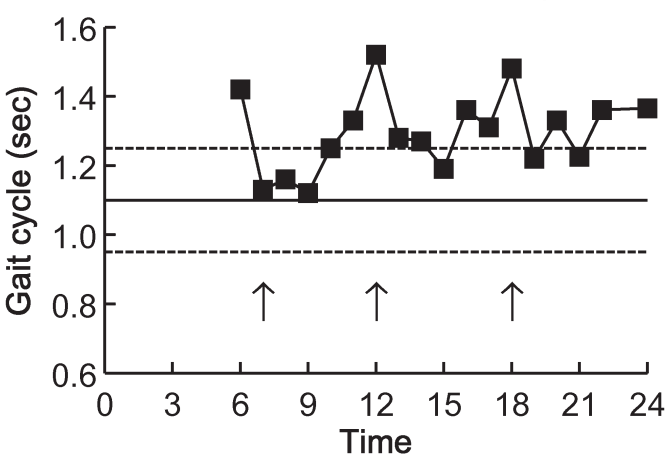

Figure 4. (A) Gait cycle in a normal control during daily activities. (B-D) Clinical classification based on gait rhythms. While some patients walked with a normal gait cycle (B, Well-controlled), others showed motor fluctuation. In the Festination to faster cycle group (C), the gait cycle was shortened due to short-stepping gait, festination, or freezing of gait, whereas in the Festination to slower cycle group (D), the gait cycle was prolonged due to gait bradykinesia or instability. Data shown in A, B, C, and D are mean \pm 2 SD. Arrows in B, C, and D indicate the time of treatment with dopaminergic agents.

The gait cycle, the duration of one gait cycle, was compared. In patients with subjectively good control, the PGR showed a normal gait cycle during walking throughout the day (Fig. 4B) (n=4, Patients 1-4, Well-controlled, see the record of Patient 1). In contrast, when patients were aware of motor fluctuation, the PGR exhibited steep corresponding changes in the gait cycle during the day. Different fluctuations were observed; a shift to a faster gait cycle was noted in patients with short-stepping walking accompanied with festination or FOG (Fig. 4C) (n=9, Patients 5-13; Fluctuation to faster cycle, see a record of Patient 6), whereas a shift to a slower gait cycle was noted in patients with bradykinesia or instability ( $\mathrm{n}=9$, Patients 14-22, Fluctuation to slower cycle, see a record of Patient 19) (Fig. 4D). In each patient, treatment with dopaminergic compounds produced a temporary improvement in the gait rhythm to within the normal range, but the effects lasted only 1-3 hours after taking the medication. These changes in gait rhythm occurred correspondingly during the off period when the patient noticed uneasiness in walking.

\section{Two opposite alterations in gait cycle and relation- ship with clinical profile}

Patients were also classified according to the daily fluctuation in gait cycle into three groups; patients with Wellcontrolled, Fluctuation to faster cycle, Fluctuation to slower cycle (Table 1). UPDRS III was not significantly different among the three groups. Instead, the appearance of the gait disturbance correlated with changes in the gait cycle.

Patients of the Well-controlled group showed a less affected parkinsonian gait, especially Patients 1, 2, and 3. According to the clinical profiles, some patients showed festination or FOG, which did not occur in the present recording. The mean gait cycle duration throughout the day was $1.08 \pm 0.02$ seconds. There were no significant differences compared with data of normal controls.

Patients with motor fluctuations characterized by a shift to faster gait cycle showed short-stepping gait with a fast cycle and shuffling. Based on the clinical profiles, patients of this group were reported to frequently develop festination (6 of 9 patients) and FOG (4 of 9 patients). In the present study, festination was noticed in 4 patients (Patients 5, 6, 7, and 8 ) and FOG was noticed in 3 patients (Patients 5, 6, 
Table 1. Characteristics of Patients Classified according to Daily Fluctuations in the Gait Cycle

\begin{tabular}{|c|c|c|c|c|c|c|c|c|c|c|c|c|c|}
\hline $\begin{array}{l}\text { Patient } \\
\text { number }\end{array}$ & $\begin{array}{l}\text { Age } \\
\text { (yrs) }\end{array}$ & Sex & $\begin{array}{c}\text { Hoehn } \\
\text { Yahr }\end{array}$ & UPDRSIII & MF & $\begin{array}{c}\text { Delayed } \\
\text { on }\end{array}$ & $\begin{array}{c}\text { Short } \\
\text { stepping }\end{array}$ & Festination & FOG & Brady & Instability & $\begin{array}{c}\text { Mean } \\
\text { cycle }(\mathrm{sec})\end{array}$ & $\begin{array}{l}\text { Deviated } \\
\text { cycle }(\mathrm{sec})\end{array}$ \\
\hline \multicolumn{14}{|c|}{ Well controlled } \\
\hline 1 & 71 & $\mathrm{~F}$ & I & 12 & - & - & - & + & - & 1 & 0 & 1.13 & \\
\hline 2 & 60 & $\mathrm{~F}$ & II & 13 & - & - & + & - & - & 1 & 1 & 1.05 & \\
\hline 3 & 66 & M & III & 14 & - & - & + & + & - & 1 & 0 & 1.09 & \\
\hline 4 & 81 & $\mathrm{M}$ & IV & 42 & - & - & + & + & + & 3 & 3 & 1.11 & \\
\hline \multicolumn{14}{|c|}{ Fluctuation to faster cycle } \\
\hline 5 & 72 & $\mathrm{~F}$ & II & 23 & + & + & + & + & + & 1 & 0 & 1.07 & 0.65 \\
\hline 6 & 63 & M & III & 36 & + & - & + & + & + & 1 & 2 & 0.92 & 0.71 \\
\hline 7 & 49 & $\mathrm{~F}$ & II & 10 & + & + & + & + & - & 1 & 0 & 1.05 & 0.71 \\
\hline 8 & 87 & $\mathrm{~F}$ & IV & 37 & & - & + & + & + & 3 & 4 & 0.97 & 0.78 \\
\hline 9 & 65 & $\mathrm{M}$ & II & 14 & - & - & + & + & - & 1 & 0 & 0.94 & 0.89 \\
\hline 10 & 40 & M & II & 10 & - & + & + & - & - & 1 & 0 & 1.01 & 0.91 \\
\hline 11 & 64 & $\mathrm{~F}$ & II & 15 & - & - & + & + & + & 1 & 1 & 0.99 & 0.92 \\
\hline 12 & 57 & $\mathrm{~F}$ & II & 23 & + & - & + & - & - & 1 & 1 & 1.01 & 0.92 \\
\hline 13 & 70 & $\mathrm{~F}$ & II & 13 & - & - & + & - & - & 1 & 0 & 0.97 & 0.93 \\
\hline \multicolumn{14}{|c|}{ Fluctuation to slower cycle } \\
\hline 14 & 82 & $\mathrm{~F}$ & III & 11 & + & - & + & - & - & 2 & 2 & 1.18 & 1.25 \\
\hline 15 & 81 & M & IV & 49 & + & + & + & - & - & 3 & 3 & 1.22 & 1.30 \\
\hline 16 & 65 & M & III & 34 & - & - & + & + & + & 3 & 2 & 1.25 & 1.35 \\
\hline 17 & 75 & $\mathrm{~F}$ & III & 27 & - & - & + & - & - & 2 & 2 & 1.29 & 1.37 \\
\hline 18 & 72 & $\mathrm{~F}$ & III & 24 & + & - & + & - & - & 2 & 2 & 1.31 & 1.37 \\
\hline 19 & 75 & $\mathrm{~F}$ & IV & 23 & + & + & + & + & - & 3 & 2 & 1.32 & 1.41 \\
\hline 20 & 64 & M & III & 36 & + & - & + & + & + & 3 & 2 & 1.41 & 1.49 \\
\hline 21 & 60 & M & III & 43 & + & - & + & - & - & 3 & 4 & 1.39 & 1.56 \\
\hline 22 & 82 & $\mathrm{~F}$ & IV & 23 & - & - & + & + & - & 2 & 2 & 1.57 & 1.72 \\
\hline
\end{tabular}

Hoehn Yahr: Hoehn and Yahr stage, UPDRS; Unified Parkinson's Disease Rating Scale, MF; motor fluctuation, Short stepping; short stepping gait, FOG; freezing of gait, Brady; UPDRS score 31, Instability; UPDRS score 30, Mean cycle; the mean of the gait cycle throughout the day, Deviated cycle; the value of the most deviated averaged gait cycle per hour from the normal range (mean $\pm 2 \mathrm{SD}$ ). Patients are arranged according to the deviated cycle.

A

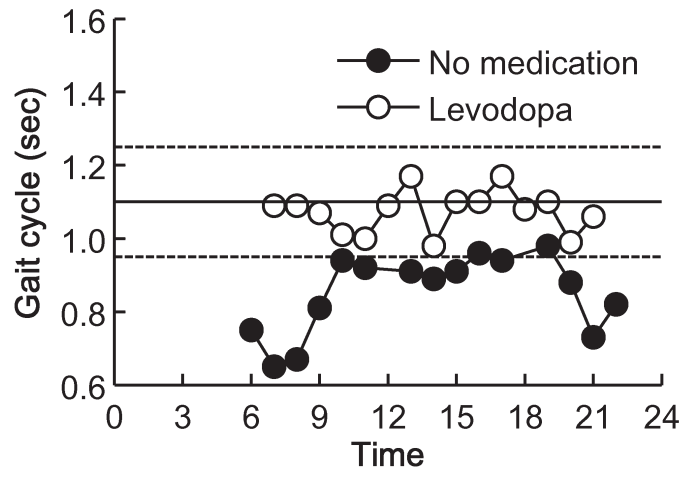

B

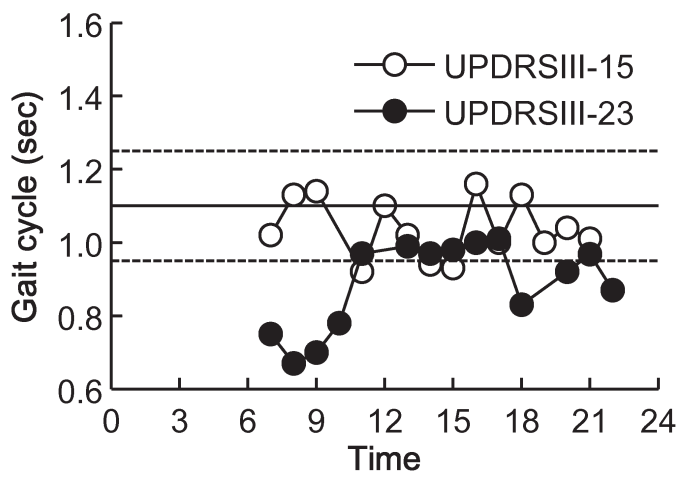

Figure 5. (A) Gait cycle patterns before and after treatment with levodopa. (B) Gait cycle changes accompanied with advancement of PD symptoms. Data are mean $\pm 2 S D$. UPDRS: Unified Parkinson's Disease Rating Scale

and 11). The mean gait cycle duration throughout the day was $0.99 \pm 0.06$ seconds, which was faster than that of the normal controls $(\mathrm{p}<0.01)$.

In contrast, patients who walked with a slower cycle during off time showed marked bradykinesia and instability. Their short-stride walking was accompanied by slowness and poverty of movements. The sum of UPDRSIII items 30

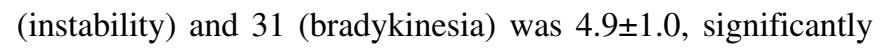
worse than that of patients of the Well-controlled group $(3.0 \pm 3.6, \mathrm{p}<0.05)$ and patients with Fluctuation to faster cy- cle $(2.1 \pm 1.9, \mathrm{p}<0.01)$. Based on the clinical profiles, festination occurred in 4 of 9 patients and FOG occurred in 2 of 9 patients. In the present study, however, none of these patients noticed evident festination or FOG. The mean gait cycle was $1.32 \pm 0.14$ seconds, which was slower than that of the normal controls $(\mathrm{p}<0.01)$.

\section{Quantitative estimation of medication effects or worsening}

We examined the effects of dopaminergic medications 
(Fig. 5A). Before the medication, one patient, a 72-year-old woman (Patient 5), had a typical parkinsonian short-step gait (UPDRS motor score 'on' state: 23). The PGR showed shortening of the mean duration of the gait cycle (0.6-0.8 seconds), particularly in the morning and evening. After treatment with levodopa, she noticed easiness in stepping and the UPDRS motor improved to 10. Consistent with these improvements, the PGR also demonstrated a normal gait cycle during walking throughout the day. In three other patients, a similar agreement between clinical symptoms and PGR findings was observed in pre- and post-medication.

In contrast, we also identified worsening of gait in other patients. For example, a 64-year-old woman (Patient 11) showed good control by levodopa, dopamine agonist and MAO-B inhibiter (UPDRS motor score 'on' state 15). PGR recording showed that the gait cycle was within the normal range throughout the day (Fig. 5B). Four months after the first recording, she frequently showed short-step gait and UPDRS motor worsened to 23. Consistent with worsening of parkinsonian gait, the PGR demonstrated shortening of the mean duration of one gait cycle (0.6-0.8 seconds). These findings indicate 'delayed-on' in the morning and 'wearing off' in the evening.

\section{Discussion}

The new PGR device allows quantitative, continuous (24 hours), and convenient recording of gait rhythm during daily activities of PD patients. PGR detected characteristic changes in walking of PD patients, but not in that of controls. Two types of changes in gait cycle occurred during off time and in parallel with the clinical severity of PD; a shift to a faster cycle and that to a slower cycle.

The alteration toward faster rhythm was observed mainly in patients who walked with short-stepping, festination and FOG. Each of the characteristic gaits could cause shortening of the gait cycle duration. Patients walking with short stride have to increase cadence compensatively, resulting in fast gait rhythm $(12,13)$. Festination was also characterized by gradual shortening of one gait cycle, whereas FOG was defined as a grouped repetition with a high frequency during shuffling trembling as documented by Ueno et al (17). In contrast, a shift to a slower rhythm was observed in patients suffering from marked bradykinesia and instability. Although some patients showed festination or FOG, the averaged per hour cycle was prolonged due to the persistent gait hypokinesia. Thus, prolongation of the stepping cycle appeared to be the result of two deficits; 1) marked slowness in swing of the lower extremities and 2) protective walking to compensate for marked instability.

PGR appears to define these two opposite pathophysiological changes, fast cycle-related or slow cycle-related pathomechanisms. On the other hand, the gait stridemonitoring device (16) does not differentiate each type, since walking with short-stride constantly occurs in all PD patients (15). Such a rhythm disorder clarified in this re- cording might underlie primarily parkinsonian motor complications $(10,17,18)$. Nakamura et al (19) reported that the frequency of finger tapping abruptly jumps to $4-5 \mathrm{~Hz}$, compared with that of resting tremor. In FOG, the gait cycle similarly converges to the frequency of $4-5 \mathrm{~Hz}$ and at such a high frequency, the production of effective floor reaction forces is impossible, resulting in retardation of forward propelling (17).

Gait movement is a sequenced automatic movement. For exact estimation of the motor deficits, it is desirable to quantify together the degree of akinesia and bradykinesia in voluntary movements. However, alteration of gait rhythms, shortening or prolongation of one gait cycle, appears to be a convenient and sensitive marker of the proper or compensatory changes underlying PD disabilities, because gait disturbance reflects an important aspect of complex motor symptoms. Characterization of motor fluctuation using the gait rhythmogram could help in a more objective selection of anti-PD medications for independent patients capable of walking unaided.

\section{References}

1. Yanagisawa N. Natural history of Parkinson's disease: From dopamine to multiple system involvement. Parkinsonism Relat Disord 12: S40-S46, 2006.

2. Marsden CD, Parkes JD, Quinn N. Fluctuations of disability in Parkinson's disease: clinical aspects. In: Movement Disorders. Marsden CD, Fahn S, Eds. Butterworth Scientific, London, 1982: 96-122.

3. Stacy M, Bowron A, Guttman M, et al. Identification of motor and nonmotor wearing-off in Parkinson's disease: Comparison of a patient questionnaire versus a clinician assessment. Mov Disord 20: 726-733, 2005.

4. Giladi N. Medical treatment of freezing of gait. Mov Disord 23: 482-488, 2008.

5. van Hilten JJ, Middelkoop HA, Kerkhof GA, Roos RA. A new approach in the assessment of motor activity in Parkinson's disease. J Neurol Neurosurg Psychiatry 54: 976-979, 1991.

6. Saito N, Yamamoto T, Sugiura Y, Shimizu S, Shimizu M. Lifecorder: a new device for the long-term monitoring of motor activities for Parkinson's disease. Intern Med 43: 685-692, 2004.

7. Hoff JI, van den Plas AA, Wagemans EA, van Hilten JJ. Accelerometric assessment of levodopa-induced dyskinesias in Parkinson's disease. Mov Disord 16: 58-61, 2001.

8. Hoff JI, van den Meer V, van Hilten JJ. Accuracy of objective ambulatory accelerometry in detecting motor complications in patients with Parkinson's disease. Clin Neuropharmacol 27: 53-57, 2004.

9. Keijsers NL, Horstink MW, Gielen SC. Ambulatory motor assessment in Parkinson's disease. Mov Disord 21: 34-44, 2006.

10. Maruyama T, Yanagisawa N. Cognitive impact on freezing of gait in Parkinson's disease. Parkinsonism Relat Disord 12: S77-S82, 2006.

11. Schaafsma JD, Balash Y, Gurevich T, Bartels AL, Hausdorff JM, Giladi N. Characterization of freezing of gait subtypes and the response of each to levodopa in Parkinson's disease. Eur J Neurol 10: 391-398, 2003.

12. Morris ME, Iansek R, Matyas TA, Summers JJ. The pathogenesis of gait hypokinesia in Parkinson's disease. Brain 117: 1168-1181, 1994.

13. Morris ME, Iansek R, Matyas TA, Summers JJ. Ability to modulate walking cadence remains intact in Parkinson's disease. J Neu- 
rol Neurosurg Psychiatry 57: 1532-1534, 1995.

14. Morris ME, Iansek R, Matyas TA, Summers JJ. Stride length regulation in Parkinson's disease. Normalization strategies and underlying mechanisms. Brain 119: 551-568, 1996.

15. Mitoma H, Hayashi R, Yanagisawa N, Tsukagoshi H. Characteristics of parkinsonian and ataxic gaits: a study using surface electromyograms, angular displacements and floor reaction forces. $\mathbf{J}$ Neurol Sci 172: 22-39, 2000.

16. Moore ST, MacDougall HG, Gracies JM, Cohen HS, Ondo WG. Long-term monitoring of gait in Parkinson's disease. Gait Posture
26: 200-207, 2007.

17. Ueno E, Yanagisawa N, Takami M. Gait disorders in parkinsonism. A study with floor reaction forces and EMG. Adv Neurol 60: 414-418, 1993.

18. Freund HJ. Motor dysfunctions in Parkinson's disease and premotor lesions. Eur Neurol 29(suppl.1): 33-37, 1989.

19. Nakamura R, Nagasaki H, Narabayashi H. Arrhythmokinesia in parkinsonism. In: Advances in Parkinsonism. Birkmayer W, Hornkievicz O, Eds. Roche, Basel, 1976: 258-268.

(C) 2010 The Japanese Society of Internal Medicine http://www.naika.or.jp/imindex.html 